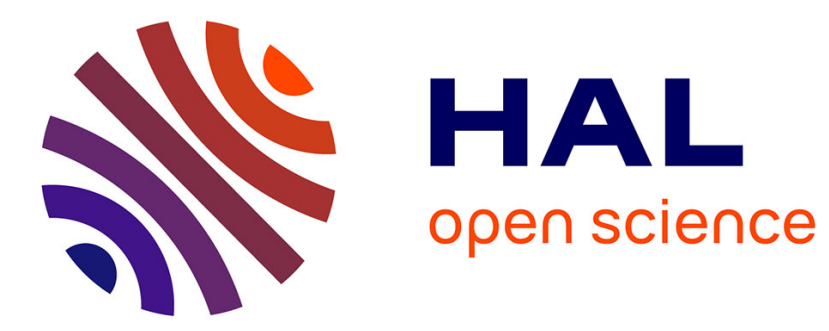

\title{
Quasi-linear approach : exact solution for nonlinear Green function
}

P. Sakov

\section{To cite this version:}

P. Sakov. Quasi-linear approach: exact solution for nonlinear Green function. Journal de Physique IV Proceedings, 1994, 04 (C5), pp.C5-833-C5-836. 10.1051/jp4:19945180 . jpa-00252862

\section{HAL Id: jpa-00252862 https://hal.science/jpa-00252862}

Submitted on 1 Jan 1994

HAL is a multi-disciplinary open access archive for the deposit and dissemination of scientific research documents, whether they are published or not. The documents may come from teaching and research institutions in France or abroad, or from public or private research centers.
L'archive ouverte pluridisciplinaire HAL, est destinée au dépôt et à la diffusion de documents scientifiques de niveau recherche, publiés ou non, émanant des établissements d'enseignement et de recherche français ou étrangers, des laboratoires publics ou privés. 


\title{
Quasi-linear approach: exact solution for nonlinear Green function
}

\author{
P.V. SAKOV
}

N.N. Andreev Acoustics Institute, Moscow 117036, Russia

\begin{abstract}
A formal way to obtain a recently found solution of the problem of nonlinear interaction of two non-concentric spherical waves in terms of quasi-linear approach is described.
\end{abstract}

\section{INTRODUCTION}

The problem of nonlinear interaction of two non-concentric spherical waves $p_{1}$ and $p_{2}$

$$
p_{1}=A_{1} \frac{\exp \left(i k_{1}\left|r-r_{10}\right|\right)}{k_{1}\left|r-r_{10}\right|}, \quad p_{2}=A_{2} \frac{\exp \left(i k_{2}\left|r-r_{20}\right|\right)}{k_{2}\left|r-r_{20}\right|} \text {, }
$$

in an ideal boundless liquid is considered below in terms of quasilinear approach. Here $\mathrm{k}_{1}$ and $\mathrm{k}_{2}$ are the wave numbers at the corresponding frequencies; the time factor of the form $\exp (-i \omega t)$ is omitted everywhere.

A solution of this problem, apart from the physical importance, may be used formally as an analog of the ordinary (linear) Green function in calculations of the secondary sound field. Namely.

Usually, it is a primary fields product in a point that is considered as an elementary secondary field source. Following this approach, to calculate the secondary sound field, one has to integrate the primary fields product along with the Green function of Helmholtz equation over the whole space. From the other hand, each of the primary fields may be presented as arisen from the primary fields sources. By changing the order of integration, one comes to an alternate representation for the secondary field. The pairs of the primary fields elementary (point) sources are treated as the secondary field elementary sources in it, and in integration over all possible combinations of these pairs, a three-point function is used. This function (called here the Nonlinear Green function) up to a constant multiple coinsides with the solution of the problem in hand.

Generally, the use of the Nonlinear Green function should be more effective than the usual integration over the whole space because it already contains effects of spatial accumulation (provided that an expression for it will turn out not too complicated). The main problem here is that the whole approach is connected with the free-space formulation. Hence, it can not be used directly if the boundary conditions or diffraction are to be taken into account. 


\section{FORMULATION}

We restrict our consideration by solution of the westervelt equation for the sum frequency field $P_{+}$,

$$
\left(\Delta+k_{+}^{2}\right) p_{+}=\beta k_{+}^{2} \frac{p_{1} p_{2}}{\rho c^{2}}
$$

where $k_{+}$is a wave number at sum frequency, $\beta$ - coefficient of nonlinearity, $\rho$ - density, $c$ - speed of sound. To turn into difference frequency field, it is sufficient to let $k_{+} \rightarrow k_{-}, k_{2} \rightarrow-k_{2}^{*}$. By knowing a solution of Eq.(2), one may easily obtain a full solution for the secondary sound field [1]. As these solutions differ only by non-cumulative terms having an explicit form, a solution of Eq. (2) for $p_{+}$(or $p_{-}$) may be considered only.

The solution of $\mathrm{Eq}(2)$ may be written as

(3) $p_{+}=-A \iiint \frac{\exp \left(i k_{1}\left|r^{\prime}-r_{10}\right|\right)}{\left|r^{\prime}-r_{10}\right|} \frac{\exp \left(i k_{2}\left|r^{\prime}-r_{20}\right|\right)}{\left|r^{\prime}-r_{20}\right|} \frac{\exp \left(i k_{+}\left|r^{\prime}-r\right|\right)}{4 \pi\left|r^{\prime}-r\right|} d^{3} r^{\prime}$,

$A \equiv \beta k_{+}^{2} A_{1} A_{2} / k_{1} k_{2} \rho c^{2}$. The problem is to obtain an expression for this integral.

\section{SOLUTION}

Recently [2], a solution of $\mathrm{Eg} \cdot(2)$ was found:

(4a)

$$
\begin{aligned}
& p_{+}=f(R)+f(-R), \\
& f(R) \equiv B \frac{\exp (i R)}{R}\left\{E i\left[i\left(R_{+}-R\right)\right]-E i\left[i\left(R_{1}-R\right)\right]-E i\left[i\left(R_{2}-R\right)\right]\right\}
\end{aligned}
$$

(4c) $\mathrm{R} \equiv\left|\mathrm{k}_{1} \mathrm{r}_{1}+\mathrm{k}_{2} \mathrm{r}_{2}\right|, \quad \mathrm{R}_{+} \equiv \mathrm{k}_{1} \mathrm{r}_{1}+\mathrm{k}_{2} \mathrm{r}_{2}, \quad \mathrm{R}_{1} \equiv \mathrm{k}_{+} \mathrm{r}_{1}+\mathrm{k}_{2} a, \quad \mathrm{R}_{2} \equiv \mathrm{k}_{+} \mathrm{r}_{2}+\mathrm{k}_{1} a$,

$$
r_{1} \equiv\left|r-r_{10}\right|, \quad r_{2} \equiv\left|r-r_{20}\right|, \quad \alpha \equiv\left|r_{10}-r_{20}\right|, \quad r_{1} \equiv\left|r_{1}\right|, \quad r_{2} \equiv\left|r_{2}\right|,
$$

where $B \equiv i A / 2$ and $E i(x)$ is the integral exponent. This solution fits $\mathrm{Eq} \cdot(2)$ provided

$$
k_{+}=k_{1}+k_{2}
$$

which is somewhat wider then the case of an ideal liquid because it allows a dissipation linearly changing with frequency. But physically this extension does not have much sense.

A solution for the difference frequency field has the same form except the formal changes mentioned above.

Although expression (4) fits Eq.(2) and has the necessary behavior at infinity (i.e., the incoming waves amplitude decrease as 
$\mathrm{r}^{-2}$ ), it is not clear how it may be proved that it really coincides with integral (3). Actually, imagine that two incoming waves sources are presented in forming solution (4), with the fields of these sources cancell each other in the main order at infinity. Then the incoming field will also behave as $r^{-2}$ at infinity, and at the same time such solutions will be not suitable. in the case

Recently, a formal way for calculating the integral (3) was found

$$
\operatorname{Re}\left(k_{+}\right)=\operatorname{Re}\left(k_{1}\right)+\operatorname{Re}\left(k_{2}\right) ; \quad \operatorname{Im}\left(k_{1}\right)=\varepsilon \operatorname{Re}\left(k_{1}\right), \quad i=1,2,3, \quad \varepsilon>0,
$$

which proves that Eq.(4) is really a stated solution of the problem involved. The principal points of this technique are described below.

\section{TECHNIQUE: PRINCIPAL POINTS}

(a) The first step in calculation of the integral (3) is the use of the integral representation

$$
\frac{\exp \left(i k_{1}\left|r-r_{1}\right|\right)}{\left|r-r_{1}\right|}=\frac{i}{2} \int_{\infty \exp (i \pi)}^{\infty} H_{0}^{(1)}\left(\xi_{1}\left|\vec{\rho}_{-}-\vec{\rho}_{1}\right|\right) e^{i \mu_{1}\left|z-z_{1}\right|} \frac{\xi_{1} d \xi_{1}}{\mu_{1}}, \mu_{1} \equiv\left(k_{1}^{2}-\xi_{1}^{2}\right)^{1 / 2}
$$

for the primary waves and the Green function in (3). For integration, a cylindrical coordinates system with the axis passing through the point of observation and the nearest to it of the primary waves centers is chosen. After integration with respect to $z^{\prime}$, the integral splits into three, each of them associated with one of the singularities existing in (3). As it turns out, two terms which correspond to the point of observation and the nearest source, do not converge if (5) is the case. This leads two the next step, which is

(b) the introduction of a small, "governing" dispersion:

$$
\delta=k_{+}-k_{1}-k_{2} \neq 0,|\delta| r_{1},|\delta| r_{2} \ll 1 .
$$

Such a dispersion makes both integrals existing in a classic sense. Main parts of both integrals have a logarithmic character with respect to $\delta$ and cancell each other as $\delta \Rightarrow 0$. In calculations, the condition (6) is used to specify the behavior of some analytical functions. The remaining terms are calculated with $\delta=0$. obtained:

Ultimately, a new representation for the secondary field is

$$
p_{+}=f_{+}+f_{1}+f_{2}
$$

where $f_{+}, f_{1}$ and $f_{2}$ are represented by one-dimensional integrals with infinite bounds. The last principal step is

(c) the calculation of these integrals. It is carried out by using of three first-order partial differential equations, to which the corresponding integrals are shown to satisfy: 
(10a) $k_{2} \frac{\partial f_{+}}{\partial z}-k_{1} \frac{\partial f_{+}}{\partial b}=2 \pi i \frac{e^{i k_{2} b}}{b} \frac{e^{i k_{1}\left(a^{2}+p^{2}\right)^{1 / 2}}}{\left(a^{2}+p^{2}\right)^{1 / 2}}$,

$$
k_{+} \frac{\partial f_{2}}{\partial a}-k_{1} \frac{\partial f_{2}}{\partial b}=-2 \pi i \frac{e^{i k_{3} b}}{b} \frac{e^{i k_{1}\left(z^{2}+\rho^{2}\right)^{1 / 2}}}{\left(z^{2}+\rho^{2}\right)^{1 / 2}},
$$

$$
k_{+} \frac{\partial f_{1}}{\partial a}+k_{2} \frac{\partial f_{1}}{\partial z}=2 \pi i \frac{e^{i k_{2}\left(a^{2}+p^{2}\right)^{1 / 2}}}{\left(a^{2}+p^{2}\right)^{1 / 2}}
$$

$$
\frac{e^{i k_{1}\left(z^{2}+\rho^{2}\right)^{1 / 2}}}{\left(z^{2}+\rho^{2}\right)^{1 / 2}} \frac{\left[\left(\alpha^{2}+\rho^{2}\right)^{1 / 2}+\alpha\right]\left[\left(z^{2}+\rho^{2}\right)^{1 / 2}+z\right]-\rho^{2}}{\left[\left(a^{2}+\rho^{2}\right)^{1 / 2}+\alpha\right]\left[\left(z^{2}+\rho^{2}\right)^{1 / 2}+z\right]-\rho^{2}},
$$

$b \equiv z-a, \quad r_{1}=(\rho, 0), \quad r_{2}=(0, a), \quad r=(0, z), \quad 0<a<z$. A more complicated character of the right-hand term in the last equation is connected with the chosen coordinate system (as the axis $p=0$ may pass only through two of three spherical waves centers in the integral (3)).

To find a solution for each function, a general solution of corresponding equation is constructed. Then, by demanding for solution to be zero as $r \Rightarrow \infty$, a necessary partial solution is easily evolved. The final expression coincides with Eqs.(4a)-(4d).

As the equations $(10 a)-(10 c)$ are of the first order, no problems of the kind discussed above in connection with the primary Westervelt equation arise.

\section{CONCLUSIONS}

Despite of a formal way found, many questions still remain without answers. Some of them have purely abstract character, such as whether a more simple way to calculate the integral (3) exist or what is the nature of the splitting of solution. The other question - if an exact solution / simple representation / "good" asymptotic expression may be found for a media with dissipation and/or dispersion.

The last is a problem of a considerable practical importance because it is the effect of dissipation which is principal for many emitting parametric arrays.

We have no space to discuss the behavior of solution (4) nor its possible applications. However, it worth to mention that it is in agreement with the earlier found solutions both for interaction of concentric spherical waves [1] and for interaction of plane and spherical waves [3]. It is also consistent with the asymptotic approach developed in the last decade by $J$. Naze Tjøtta and $S$. Tjøtta $[4,5]$.

\section{REFERENCES}

1. L.W.Dean, J.Acoust. Soc. Am., 34 (1962), 1039.

2. P.V. Sakov, Proc. 13th ISNA, ed. by H.Hobæk (1993), 107.

3. L.M.Lyamshev and P.V.Sakov, Sor. Phys. Acoust., 34 (1988), 281.

4. G.S.Garett, J.N.Tjøtta and S.V.Tjøtta, J.Acoust.Soc. Am., 74 (1984), 1013 .

5. J.Berntsen, J.N.Tjøtta and S.Tjøtta, J.Acoust.Soc.Am., 86 (1989), 1968. 